\title{
External Otitis
}

\author{
Dennis C Fitzgerald*, Thomas Wilcox and Rebecca Chiffer \\ Department of Otolaryngology-Head and Neck Surgery, Jefferson Balance and Hearing Center, USA.
}

*Corresponding author: Dennis C Fitzgerald, Department of Otolaryngology-Head and Neck Surgery, Jefferson Balance and Hearing Center, Thomas Jefferson University, USA.
Received Date: August 20, 2020

Published Date: September 04, 2020

\section{Short Communication}

\section{What is acute external otitis?}

(Swimmer's Ear) Acute external otitis is an infection of the ear canal caused by bacteria. The infection seldom involves the inner ear and therefore rarely leads to permanent hearing loss. It is also called 'swimmer's ear' because it is frequently due to water contamination of the ear canal, It may also be caused by picking, scratching or manipulating the ear canal with things like 0 -tips $\AA^{\circledR}$, paperclips, pens, etc.,. Frequently, pain can be severe due to swelling of the ear canal skin. Sometimes it can be so severe that the ear canal opening swells shut and it is necessary to insert a wick to keep the ear canal open.

\section{How is acute external otitis swimmer's ear treated?}

Follow three principles to help cure your external otitis:

1. Do not clean or scratch your ear canals. It is difficult to restrain yourself since the ear(s) may feel blocked or itch. However, any manipulation will prolong the pain and delay healing. Your doctor will carefully clean you ear canals of any debris that may be blocking them. Ear drops will help to relieve the itch.

2. Do not wash your ears, use hydrogen peroxide, or allow any water to run into the canal(s). The infection is usually made worse by water, so avoid it. If you must wash your hair, gently place cotton in the ear canal and seal over the cotton with Vaseline ointment Do not leave the cotton in since the humidity in your ear canal will increase and interfere with healing.

3. Use the ear drops as prescribed by your doctor. It is usually for 7-10 days This hinders recurrence by killing residual bacteria. If a wick has been put in your ear, place the drops on the wick The wick should be removed in 48 to 72 hours, If the wick falls out early, that is a good sign that the swelling is much less However, if the ear pain persists or worsens, you need to see the doctor.

Pain in the ear and neck is common and is seldom relieved for at least 48 hours after therapy has been started. Even narcotics may not relieve the pain. The use of steroids, for example, a Medral Dosepak will often relieve the pain in 2-3 hours and decrease the need for narcotic medications It works by quickly reducing the swelling in the ear canal A prescription for a pain medication is available upon request. If your outer ear(s) swell and turn red, discontinue the ear drops since you may have an allergy to one of the medicines in it. Please contact this office immediately for a change of therapy.

\section{How is external otitis prevented?}

After healing is completed, swimming and other water exposure is allowed. If your doctor says that it is okay, and you do not have a perforated eardrum, you can make your own ear drops by evenly mixing white vinegar and rubbing alcohol. Several drops of this mixture should be placed in the ear canals after water exposure. Additionally, a portable hair dryer on a medium setting and held at a distance may be applied to the ears to hasten drying. Never stick 0 -tips ${ }^{\circledR}$ or other objects in your ear canals to dry them.

\section{Follow-up}

Please make sure that you have an appointment to return in one to three weeks for a final check and review of your condition. 


\section{Fungal external otitis}

Fungal External Otitis is the cause of external ear infections in $10 \%$ of cases. The most common fungus is Aspergillus, which produces a gray-black residue in the ear canal. The most common symptoms are: itching, swelling, moderate pain and hearing loss, most cases of Fungal External Otitis occur in the presence of a chronic inflammation of the ear canal skin, e.g. seborrhea. Seborrhea, commonly known to cause dandruff, often causes itching and flaking skin at the opening of the ear canal. With trauma induced by scratching the ear canal skin, the fungi take over and create an infection.

\section{How is fungal external otitis treated?}

Initial treatment is a thorough cleaning of the ear canal, followed by medication specifically meant to treat fungal infections, This can be administered by powder, creams or drops. Usually a fungal infection needs to be treated for three weeks to accomplish complete eradication of the infection.

\section{Chronic external otitis}

As mentioned in the explanation of fungal external otitis, seborrhea or occasionally psoriasis can cause itching and scabbing of the opening of the ear canal. It can also occur around the outside of the ear and even on the face. Recurrent treatment of the affected areas can often prevent bacterial and fungal infections from developing. These periods of itching and flaking occur randomly when the condition flares up. A twice daily application of cortisone cream or drops for one week at a time will stop the itching and flaking. These flare ups will continue to occur and require local steroids until the dermatologists find a cure for this distressing and persistent dermatitis (inflammation of the skin).

\section{Acknowledgement}

None.

\section{Conflict of Interest}

No conflict of interest. 